\title{
Nuclear physics and astrophysics experiments at ELI-NP: The emerging future
}

\author{
Dimiter L. Balabanski ${ }^{1, *}$ \\ ${ }^{1}$ Extreme Light Infrastructure - Nuclear Physics, Horia Hulubei National Institute for R\&D in Physics \\ and Nuclear Engineering, Reactorului Str. 30, 077125 Bucharest - Magurele, Romania
}

\begin{abstract}
The status of implementation of the ELI-NP high-power laser system and the high-brilliance gamma beam system is reported. The emerging experimental program at the facility in nuclear physics in astrophysics is discussed, with emphasis of the considered day-one experiments.
\end{abstract}

\section{The ELI-NP Facility}

The main research tools at Extreme Light Infrastructure Nuclear Physics (ELI-NP) facility are a high-power laser system (HPLS) and a high-brilliance gamma-beam system (GBS) [1]. They are superior to what is available at present in research laboratories worldwide. The mission of the facility, which will become operational in 2019 as an open access user facility, is cutting-edge research in the field of nuclear photonics. The HPLS has central wavelength of $815 \mathrm{~nm}$, reaching $10 \mathrm{PW}$ peak power in pulses of duration below $22 \mathrm{fs}$, with a contrast of $10^{13}: 1$ at 100 ps and $10^{9}: 1$ at 10 ps ahead of the pulse peak. It has two laser arms, operated by a common front-end. Each laser arm has three optical compressors, at $10 \mathrm{PW}, 1 \mathrm{PW}$ and $100 \mathrm{TW}$. The $10 \mathrm{PW}$ outputs can provide pulses once per minute, the $1 \mathrm{PW}$ outputs operate at $1 \mathrm{~Hz}$ and the $100 \mathrm{TW}$ outputs - at $10 \mathrm{~Hz}$. The two arms will be synchronized and experiments with any combination of pulses will be possible $[2,3]$. The HPLS system and the support target laboratory are placed in clean room areas.

The ELI-NP GBS will provide beams in the range between $200 \mathrm{keV}$ and $19.5 \mathrm{MeV}$ with a bandwidth better than $0.5 \%$, spectral density of about $10^{4}$ photons $/(\mathrm{eV} \cdot \mathrm{s})$ and linear polarization higher than $95 \%$. The gamma beam will be produced by inverse Compton scattering of $515 \mathrm{~nm}$ green laser light, provided by $100 \mathrm{~Hz} \mathrm{Yb:YAG} \mathrm{lasers,} \mathrm{off} \mathrm{electron}$ beams accelerated to relativistic energies by a warm linac consisting of S-band photoinjector and C-band acceleration cavities [4]. The electrons will interact with the laser beams at two interaction points, at low and high electron beam energies, producing low (200 keV to $3.5 \mathrm{MeV}$ ) and high energy ( $3 \mathrm{MeV}$ to $19.5 \mathrm{MeV}$ ) gamma beams. The gamma beams will be delivered along two beam lines, placed after each interaction point. The electron beam will be bunched with a frequency of $100 \mathrm{~Hz}$, and each bunch will consist of a train of 32 microbunches. Laser re-circulators will be used at the interaction points to

\footnotetext{
*Corresponding author: dimiter.balabanski@eli-np.ro
} 
ensure the interaction of the laser pulse with each bunch in the train. The gamma-beams will be collimated, cutting the low energy $\gamma$-rays, for achieving narrow bandwidth.

The ELI-NP HPLS and GBS are placed on an anti-vibration slab. The latter is separated from the side walls and the roof of the laboratory and it is placed on a system of springs and dampers, mounted on support columns in the basement of the laboratory. The whole structure is designed as a 150,000 ton optical table. It is specified to have the power spectral density of the vibrations at the level of $10^{-10} \mathrm{~g}^{2} / \mathrm{Hz}$ in a frequency range between $1 \mathrm{~Hz}$ and $200 \mathrm{~Hz}$. With this parameter achieved, the expected pointing fluctuations of the system shall remain below $3 \mu \mathrm{rad}$ root mean square. For avoiding the long-term drift of the pointing, the temperature in the laboratory building is kept at $22{ }^{\circ} \mathrm{C}$, stabilized to $\pm 0.5{ }^{\circ} \mathrm{C}$, which is achieved by a $5 \mathrm{MW}$ cooling/heating system based on geothermal water. The water is provided from a water layer at $120 \mathrm{~m}$ depth through 1000 wells, which are dig within the territory of the lab. After processing, the water is pumped back in the underground water layer. The building was commissioned in September 2016 and the measurements confirmed the stability of the floor and the temperature.

Several types of experiments will be possible at ELI-NP, e.g. laser-driven experiments in single or double pulse-shot mode on target, gamma-beam experiments in narrow- or wide-bandwidth mode, and combined laser- and gamma-beam experiments. Thus, the ELINP laboratory opens a new dimension for nuclear physics studies with intense electromagnetic probes. The experimental program, which is under preparation at ELI-NP, targets all these experimental modes and a large variety of instruments are under construction for its realization $[2,5,6]$. In this paper the emerging experimental program in the field of nuclear physics in astrophysics is described, with an emphasis of the considered day-one experiments.

\section{Nuclear physics in astrophysics research at the ELI-NP HPLS}

The availability of high-power laser pulses with intensities reaching $10^{23} \mathrm{~W} / \mathrm{cm}^{2}$, which corresponds to light pressures of $10^{13}$ Tor, is expected to open new regimes for laser-driven particle acceleration. Day-one experiments, after commissioning the laser beams, will target proton acceleration up to $200 \mathrm{MeV}$ through laser-matter interaction on $\leq 1 \mu \mathrm{m} \mathrm{CH}$ targets. On a longer term the program will address acceleration of dense ion beams on targets of increasing $Z$, aiming at accelerating actinide beams. It is expected that at such extreme light pressures, the radiation pressure acceleration (RPA) mechanism will become possible. Solid targets, which are foils of nanometre thickness, will be used in experiment. There, as a result of the laser-matter interaction, cold electron compression is achieved in front of the laser pulse, which accelerates the ions via the created dipole electrostatic field. In this way, solid-state density ion bunches, scaling with the Avogadro number, e.g. beams of up to $10^{22}$ ions/pulse are formed [7], many orders of magnitude more dense then the bunches provided by present-day accelerators. Thus, due to the light pressure, the part of the foil which is hit by the laser pulse is accelerated as a whole, like a "light sail", and a good fraction of the laser energy is converted in accelerating ions up to $\mathrm{GeV}$ energies.

The nuclear physics program at the ELI-NP HPLS addresses studies of nuclear reactions of astrophysics interest in a controlled plasma environment, which will help understanding the screening effect, i.e. the reaction cross section variation due to the presence of the electron cloud around nuclei in hot plasma conditions [8]. One laser beam will be used to create a secondary plasma target with controlled temperature and density, while another laser pulse will accelerate ions from a primary target that will impinge on short time scale, up to $1 \mathrm{ps,} \mathrm{on} \mathrm{the} \mathrm{rapidly} \mathrm{expanding} \mathrm{plasma} \mathrm{target.} \mathrm{Plasma} \mathrm{environment} \mathrm{is} \mathrm{expected} \mathrm{to}$ produce changes also in other nuclear observables, such as decay modes and half-lives of unstable states. Large number of free electrons, photons and highly charged ions 
encountered in high-temperature high-density plasmas may induce nuclear excitations and de-excitations at high rate, which become possible through new mechanisms, such as nuclear excitation through electron capture (NEEC) or through electron transition (NEET), schematically shown in Fig. 1. The realization of these processes in hot plasmas might result in changes by many orders of magnitude in half-lives of some states, as have been estimated for ${ }^{93 \mathrm{~m}} \mathrm{Mo}$ and ${ }^{26 \mathrm{gs}} \mathrm{Al}$ [8], which are considered as candidates for day-one studies.

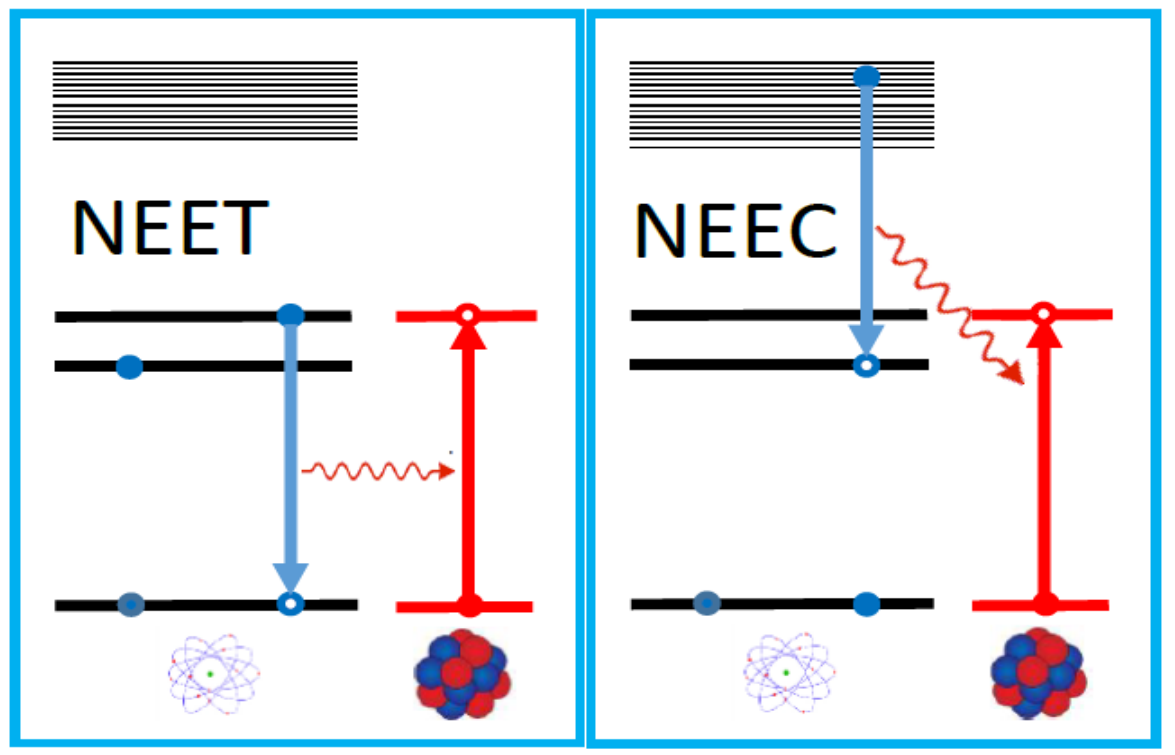

Fig. 1. (colour online) Schematic presentation of the nuclear excitation through electron transition (NEET) process (left) and the nuclear excitation through electron capture (NEEC) process (right).

While indications of the NEET process have been reported in cold targets at $10^{-8}$ rates in ${ }^{197} \mathrm{Au},{ }^{189} \mathrm{Os}$, and ${ }^{183} \mathrm{Ir}$ [9-11], experimental observations of the NEEC process have never been reported.

On a different note, the origin of the screening effect has been disputed a lot over the last 20 years in an attempt to understand the need of exceedingly high values of the screening potential needed to explain the data. Recently it was suggested that the reason for the large screening potential values is in fact due to clusterization effects in nuclear reactions, in particular for reaction involving light nuclei [12]. Experiments at ELI-NP will shed light on this issue.

On a longer term, this program aims at acceleration of dense beams of actinide nuclei at energies above the Coulomb barrier for nuclear reactions, and investigation of the mechanism of the fission-fusion reaction [13]. In the first stage of the reaction, the accelerated actinide nuclei impinge on a secondary target and fission. However, the density of fission fragments is large enough to result in a subsequent fusion. The fusion of light fission fragments would provide a possibility for studies of the structure of nuclei in the vicinity of the $N=126 r$-process waiting point.

\section{Nuclear physics in astrophysics research at the ELI-NP GPS}

At the ELI-NP GBS the probe for nuclear studies is a photon with well-defined energy and polarization. A number of photonuclear reactions will be possible with these gammabeams, which are displayed schematically in Fig. 2, namely nuclear resonance fluorescence 
(NRF) studies, photo-neutron reactions and photo-charged-particle reactions, studies of large collective motions in nuclei, photo-activation and photo-fission.

The availability of high-brilliance, narrow-bandwidth, pencil-size, polarized ELI-NP beams will result in unique NRF measurements [14]. A most appealing case, which can be done at the low-energy gamma beam line already in the commissioning phase, is the photoactivation measurement of ${ }^{180 \mathrm{~m}} \mathrm{Ta}$, i.e., a measurement of the subsequent $\gamma$ decay as a function of the gamma-beam energy. Doorway states in ${ }^{180 \mathrm{~m}} \mathrm{Ta}$, through which the $I^{\pi}=9^{-}$, $\mathrm{T}_{1 / 2}>1.2 \cdot 10^{15}$ year isomer is de-excited, have been studied in bremsstrahlung experiments [15]. This experiment is closely related to nuclear astrophysics research, since the detailed knowledge of the doorway states and the flux which passes through them, provide a sensitive thermometer for studying the star conditions during the nucleosynthesis. Later, on day-one, NRF studies with actinide targets can be performed, which become possible due to the high-brilliance and small spot size of the beam, which require milligram targets to be used.

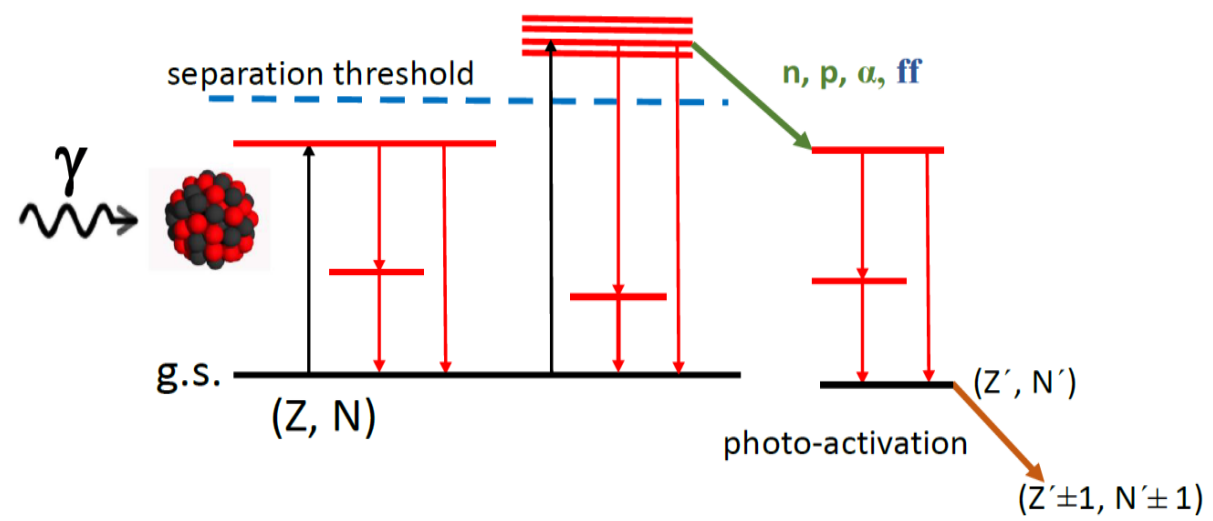

Fig. 2. (colour online) Schematic presentation of the photonuclear processes which can be studied in experiments at the ELI-NP GBS. The decay of excited states below the particle separation threshold (right) is studied with the nuclear resonance fluorescence method. Studies of the decay of excited states above the separation threshold (middle) are related to large-amplitude collective motions, which can be carried out either through neutron emission, or through gamma decay. Studies of charged-particle reactions and photo-fission are also indicated, as well follow-up photo-activation studies in daughter nuclei (right).

Studies of photo-disintegration processes at ELI-NP are related to the Big-Bang nucleosynthesis (BBN), He-burning in stars, and $p$ - and $s$ - nucleosynthesis processes. The ${ }^{7} \mathrm{Li}(\gamma, \mathrm{t}) \alpha$ reaction addresses two aspects, which need further insight, e.g. experimental data below $1.5 \mathrm{MeV}$ disagree with theoretical calculations, and measurements at higher energies can be used for restricting the extrapolation to energies relevant to astrophysics. A first measurement of this reaction has been done recently at the HI $\gamma \mathrm{S}$ gamma-beam facility at Duke University, USA [16], the continuation of this study is considered as a day-one experiment at ELI-NP.

In massive star evolution and stellar explosive sites (Type Ia, II, and Ib/c Supernovae), the astrophysical $p$-process is an important way of nucleosynthesis to produce the protonrich, stable nuclides beyond Fe which cannot be reached by the $s$ - and $r$-processes. The common picture is that these nuclides are synthesized by photodisintegration of pre-existing $s$ - and $r$-processes nuclei. The dominant nuclear flows of $p$-process go towards the neutrondeficient region through $(\gamma, n)$ reactions. At present, systematic reaction network calculations are been carried out [17], in order to define the best cases to be explored. For 
example, astrophysical simulations show that the nucleosynthesis of ${ }^{92,94} \mathrm{Mo}$ and ${ }^{96,98} \mathrm{Ru}$ are systematically under-produced by benchmarking of the predicted abundances of these nuclei to their corresponding abundances observed in solar system composition. Therefore, it is expected that the experimental determination of the astrophysical reaction rates around $\mathrm{Mo}$ and $\mathrm{Ru}$ would remedy this unsolved problem of $p$-process nucleosynthesis.

The ${ }^{16} \mathrm{O}(\gamma, \alpha){ }^{12} \mathrm{C}$ reaction, the inverse of ${ }^{12} \mathrm{C}(\alpha, \gamma){ }^{16} \mathrm{O}$ reaction, is also suggested to be studied at ELI-NP [18]. Modelling the evolution and explosion of massive stars requires a $10 \%$ uncertainty on the ${ }^{12} \mathrm{C}(\alpha, \gamma){ }^{16} \mathrm{O}$ reaction cross section, while a recent analysis of the world data estimated this uncertainty at around 20\% [19]. The goal of the measurements at ELI-NP will be to measure detailed cross sections and angular distribution of the reaction products, thus obtaining accurate values of the E2/E1 ratio. One of the advantages of measuring the photo-dissociation of ${ }^{16} \mathrm{O}$ is a gain in cross section due to the detailed balance theorem. The majority of ${ }^{12} \mathrm{C}(\alpha, \gamma){ }^{16} \mathrm{O}$ experimental and theoretical work over the last 60 years have concentrated in the energy region below $10 \mathrm{MeV}$. However, measurements at higher energies, all the way to $14 \mathrm{MeV}$, would allow to include more states in the R-matrix analysis and to reduce the uncertainty of the extrapolation at lower energies [20]. The aim of such measurements at ELI-NP would be to determine angular distributions and cross sections between known resonances with statistical precision of $3 \%$ or better. For example, a measurement of the weakest cross section between the $2^{+}$states at 9.84 and $11.52 \mathrm{MeV}$ will require twelve hours of beam time for each experimental point.

Experiments at and above the neutron separation threshold will aim at detailed studies of E1 strength in atomic nuclei [21], which will provide reliable estimates of nuclear polarizability, an observable, which some theories relate to the symmetry term of the nuclear equation of state (EoS) [22-24]. In addition, detailed studies of the gamma-decay of the GDR both to the ground and excited states will be possible, which will set constraints on different theoretical models. The ELI-NP experimental program will provide highresolution data of the distribution of the E1 and M1 strength in nuclei using a $\gamma$-ray probe, which is complimentary to the results obtained in $\left(\mathrm{p}, \mathrm{p}^{\prime}\right)$ and $\left(\alpha, \alpha^{\prime}\right)$ measurements. As a first experiment the cross section of ${ }^{9} \mathrm{Be}(\gamma, \mathrm{n})$ reaction can be studied at $\gamma$-beam energies between 1.65 MeV and 3.20 MeV. This cross section has been measured several times. However, there is considerable $(>20 \%$ ) discrepancy between the recently reported results $[25,26]$ and an independent measurement might resolve this discrepancy in early ELI-NP operation.

Another area of interest is related to neutron-capture rates, which can be determined by inverse photodisintegration and photoexcitation studies [27,28]. Such measurements will be pursued for many of the critical $s$-process branching point nuclei, which will help to determine the neutron flux and the temperature conditions at the $s$-process sites.

Finally, photo-fission experiments will aim at studies of the landscape of the threedimensional multi-humped fission barrier in the actinide nuclei. This will be done through studies of transmission resonances [29]. Within this approach, a state in the first minimum of the nuclear potential is excited and couples to states in the second or third minima of the potential barrier. This coupling results in resonance states in the fission cross section in the region $5 \mathrm{MeV}$ to $7 \mathrm{MeV}$ [30]. The experiments will aim at the characterization of these transmission resonances through the measurement of angular distributions, and the identification of the fission fragments.

\section{Conclusions}

A diverse research program, which addresses different topics of nuclear physics in astrophysics research will be carried out at ELI-NP. Cases of flagship experiments were defined, and certain low-hanging fruits, which should be measured first at the start of the 
facility, have been identified. Experiments at the ELI-NP HPLS will aim at improving our knowledge on nuclear reactions in a plasma environment and at understanding the electron screening effect and the $N=126$ waiting point of the $r$-process. Experiments at the ELI-NP GBS will shed light to open problems related to the BBN, the evolution of massive stars and explosive sites, and to a better understanding of the $s$ - and $p$-processes.

This work is supported by the Extreme Light Infrastructure Nuclear Physics (ELI-NP) Phase II, a project co-financed by the Romanian Government and the European Union through the European Regional Development Fund - the Competitiveness Operational Programme (1/07.07.2016, COP, ID 1334).

\section{References}

1. N. V. Zamfir, Nucl. Phys. News 25:3, 34 (2015).

2. 2. S. Gales et al., Phys. Scr. 91, 093004 (2016).

3. N. V. Zamfir, Eur. Phys. J. Special Topics 223, 1221 (2014).

4. O. Adriani et al., arXiv:1407.3669 [physics.acc-ph] (2014).

5. Rom. Rep. Phys. 68 Supplement, ELI-NP Technical Design Reports (2016).

6. D. L. Balabsnki et al., Europhys. Lett. 117, 28001 (2017).

7. A. Macchi, M. Borghesi, M. Passoni, Rev. Mod. Phys. 85, 751 (2013).

8. F. Negoita et al., Rom. Rep. Phys. 68, S37 (2016).

9. S. Kishimoto et al., Phys. Rev. Lett. 85, 1831 (2000).

10. I. Ahmad et al., Phys. Rev. C 61, 051304 (2000).

11. S. Kashimoto et al., Nucl. Phys. A 748, 3 (2005).

12. C. Spitaleri et al., Phys. Lett. B 755, 275 (2016).

13. D. Habs et al., Appl. Phys. B 103, 471 (2011).

14. C. A. Ur et al., Rom. Rep. Phys. 68, S483 (2016).

15. D. Belic et al., Phys. Rev. C 65, 035801 (2002).

16. C. Matei et al., Phys. Rev. C (in preparation).

17. H. Y. Lan et al., Nucl. Inst. Meth. Phys. Res. A (in preparation).

18. O. Tesileanu et al. Rom. Rep. Phys. 68, S699 (2016).

19. R. J. deBoer et al, Rev. Mod. Phys. 89, 035007 (2017).

20. D.B. Sayre et al., Phys. Rev. Lett. 109, 142501 (2012).

21. F. Camera et al., Rom. Rep. Phys. 68, S539 (2016).

22. P.-G. Reinhardt, W. Nazarewicz, Phys. Rev. C 81, 051303 (2010).

23. J. Piekarewicz, Phys. Rev. 83, 034319 (2011).

24. X. Roca-Maza et al., Phys. Rev. Lett. 106, 252501 (2011).

25. H. Unsunomiya et al., Phys. Rev. C 92, 064323 (2015).

26. C. W. Arnold et al., Phys. Rev. C 85, 044605 (2012).

27. R. Raut et al., Phys. Rev. Lett. 111, 2501 (2013).

28. A. Sauerwein et al., Phys. Rev. C 89, 035803 (2014).

29. D. L. Balabanski et al., Rom. Rep. Phys. 68, S621 (2016).

30. A. Krasznahorkay, in: Handbook of Nuclear Chemistry (Springer Verlag, Dordrecht, 2011), p. 281. 